\title{
Design and realization of a compact and multi-purpose passively Q-switched ND:YAG laser system
}

\section{Original Article}

\author{
Adel Abdallah Mohamed \\ Military Technical College
}

\section{Keywords:}

Laser realization, Nd:YAG laser, passive Q-switch, passive Q-switch modeling.

\section{Corresponding Author:}

Adel Abdallah Mohamed, Military Technical College, Cairo, Egypt, Tel: +201009872699

Email: adel.abdallah@mtc.edu.eg

\section{Abstract}

In this paper, a passively Q-switched pulsed Nd:YAG laser system is designed and realized for defense applications. Our previously published generalized mathematical model for passively Q-switched lasers is used to help extract the design parameters of the proposed Q-switched Nd:YAG laser system. The proposed laser system is a simple and compact laser source which can be employed for many applications. The laser system operates at $1064 \mathrm{~nm}$, its free running output energy is $150 \mathrm{~mJ}$, while its Q-switched laser output contains a train of pulses with pulse width varies from $47 \mathrm{~nm}$ to $85 \mathrm{~nm}$ and with maximum measured total output energy of $52.4 \mathrm{~mJ}$. The output pulse series contains 6 pulses with maximum peak power of $468 \mathrm{~kW}$, while the minimum obtained peak power is $18.8 \mathrm{~kW}$. The total duration of the Q-switched output pulse series is about $150 \mu \mathrm{s}$. This system can be used in many applications such as Laser-induced breakdown spectroscopy (LIBS), LIDAR, laser target designation (LTD), range finding, and some medical applications.

\section{INTRODUCTION}

In the past years, several developments have been made in the research of laser design field, aiming to achieve compact, cost-effective, high beam quality and electrically efficient laser systems at reduced cost that are capable of delivering high energy pulses in the order of ten nanoseconds. These kinds of high pulse energy allsolid-state lasers are widely used in scientific research, industrial, and military applications like airborne laser system, LIDAR and laser target designator ${ }^{[1]}$. The specific properties of $\mathrm{Cr}^{4+}$ :YAG crystals such as large absorption band, high damage threshold, long lifetime, good thermal stability and easy operation enabled it to be one of the best and durable passive Q-switch crystals in the $\mathrm{Nd}^{3+}$ based lasers ${ }^{[2,3]}$. Many applications such as LIDAR, and portable laser target designator require passively Q-switched $\mathrm{Nd}$ :YAG laser systems that is very compact, lightweight, efficient and versatile ; where size, weight, reliability and power are major factors. In this paper, the design and realization of a compact passively Q-switched flash lamp pumped Nd:YAG laser system for defense applications is proposed. We used our previously published modified and enhanced passively Q-switched Nd:YAG laser model presented ${ }^{[4]}$, to help design the proposed laser system. The laser system consists of a closed-coupled cavity contains a $5 \mathrm{~cm} \times 5 \mathrm{~mm} \mathrm{Nd}$ :YAG laser rod and a linear Xenon flash lamp as a pump source with pump energy of $22.5 \mathrm{~J}$ and pulse width $1 \mathrm{~ms}, 50 \%$ output coupler reflectance, a cavity length of $20 \mathrm{~cm}$, and $\mathrm{Cr}^{4+}$ :YAG as saturable absorber with initial transmission of $50 \%$ are used. We obtained a single shot Q-switched laser output that consists of a train of 6 pulses. The maximum total output pulse energy of the pulse train is $52.4 \mathrm{~mJ}$ with the main Q-switched pulse has energy of $22 \mathrm{~mJ}$, pulse width of $47 \mathrm{~ns}$ and peak power of $468 \mathrm{~kW}$.

\section{PASSIVELY Q-SWITCHED ND:YAG LASER MODELING}

Our enhanced mathematical model of the passively Q-switched Nd:YAG laser is used to help us extract the design parameters of the proposed laser system ${ }^{[4]}$, and is given by the following coupled-rate equations:

$$
\begin{aligned}
& \frac{d \varphi}{d t}=\frac{\varphi}{t_{r}}\left[2 \sigma n l-2 \sigma_{g i} n_{g 1} l_{:}-2 \sigma_{*} n_{* 1} l_{s}-2 \sigma_{*} n_{* 2} l-\left(\ln \left(\frac{1}{R}\right)+L\right)\right] \\
& \frac{d n}{d t}=P-\frac{n}{\tau_{f}}-\gamma \sigma c \varphi n \\
& \frac{d n_{e s 1}}{d t}=\sigma_{g s} c \varphi n_{g s}-\frac{n_{e s 1}}{\tau}-\sigma_{e s} c \varphi n_{e s 1}+\frac{n_{e s 2}}{\tau^{*}} \\
& \frac{d n_{g s}}{d t}=-\frac{A}{A_{s}} \sigma_{g s} c \varphi n_{g s}-\frac{n_{e s 1}}{\tau} \\
& \frac{d n_{e s 2}}{d t}=\sigma_{e s} c \varphi n_{e s 1}-\frac{n_{e s 2}}{\tau^{*}}
\end{aligned}
$$


in which $l$ is the length of the gain medium, $l_{\mathrm{s}}$ is the saturable absorber crystal thickness, $c$ is the velocity of light, $\gamma$ is the inversion reduction factor, $R$ is the output coupler reflectance, $\mathrm{L}$ is the cavity dissipative loss, ngs is the ground-state atoms density, $n_{e s 1}$ and $n_{e s 2}$ are the first and the second excited-states atoms density of the saturable absorber, respectively, $n$ is the inversion density of the laser medium, $\varphi$ is the photon density, $t_{r}$ is the round trip time, $\sigma_{\mathrm{gs}}$ and $\sigma_{\mathrm{es}}$ are the ground and excited state absorption cross-section areas of the saturable absorber, respectively, $\sigma$ is the stimulated emission cross-section area of the active medium, $\tau$ is the decay time from the first excited state to the ground state of the saturable absorber, $A / A_{s}$ is the ratio of the effective area in the gain medium and in the saturable absorber, $\tau_{\mathrm{f}}$ is the emission life time of the laser crystal, $P$ is the pump rate density in unit $\left(\mathrm{cm}^{-3} \mathrm{~s}^{-1}\right)$, and $\tau^{*}$ is the decay time from the second excited state to the first excited state of the saturable absorber. Passive Q-switching is accomplished by making the fractional loss per round trip, $L$, a function of the photon density. The losses in the cavity can be represented by, $L(t)=L_{R}+$ $L_{Q}(t)$, where the first term, $L_{R}$, is the dissipative round trip loss contains the output coupling losses determined by the mirror reflectivity $\mathrm{R}$, and all the incidental losses such as scattering, diffraction, and absorption, and the second term, $L_{Q}(t)$, represents the loss introduced by the Q-switch. The transmission of the saturable absorber is given by:

$$
T_{i}=\exp \left(-\alpha_{o}(E) l_{s}\right)=\exp \left(-n_{g s}(t) \sigma_{g s} l_{s}\right)
$$

in which $\alpha_{o}(\mathrm{E})$, is the absorption coefficient which is intensity dependent, and is given by ${ }^{[3]}$ :

$$
\alpha_{o}(E)=\frac{\alpha_{o}}{1+E_{i} / E_{s}}
$$

in which $\alpha_{o}$, is the small-signal absorption coefficient and Esis a saturation fluence which is given by ${ }^{[3]}$ :

$$
E_{s}=\frac{h v}{\sigma_{g s}}
$$

Equation (6), reduces to $\mathrm{T}_{\mathrm{o}}$, for $\mathrm{E}_{\mathrm{i}}<<\mathrm{E}_{\mathrm{s}}$, and to 1, for $\mathrm{E}_{\mathrm{i}}>>\mathrm{E}_{\mathrm{s}}$. From these equations, we concluded that the transmission of the absorber varies with time according to the relation between the incident fluence and the saturation fluence of the absorber. Accordingly, $L_{Q}(t)=2 \sigma_{e s} n_{g s}(t) l_{s}$, that represents the cavity loss introduced by the Q-switch will vary and this variation must be included in the model. Solving equations (6), (7), and (8), $\mathrm{L}_{\mathrm{Q}}$ can be expressed as:

$$
L_{Q}(t)=\frac{2 \sigma_{e s} l_{s} \alpha_{o}}{\sigma_{g s}\left(1+\left(E_{i}(t) / E_{s}\right)\right)}
$$

Thus:

$$
L(t)=L_{R}+L_{Q}(t)
$$

Replacing $L$ in equation (1) by equation (10) provides a comprehensive model that shows the same behavior as that of the real practical systems. The total energy in the Q-switched pulse can be obtained using the following relation ${ }^{[5]}$ :

$$
E=\frac{h v A l_{c}}{t_{r}} \ln \left(\frac{1}{R}\right) \int \phi(t) d t
$$

Where $A l_{c}$ is the cavity volume occupied by the photons, $t_{r}$ is the cavity round trip time, and $\mathrm{R}$ is the output coupler reflectance. This model helped us extract the design parameters of the proposed passively Q-switched $\mathrm{Nd}: Y A G$ laser system.

\section{LASER SYSTEM DESIGN AND CHARACTERIZATION}

It is required to realize a compact pulsed $\mathrm{Nd}: \mathrm{YAG}$ laser source to obtain $150 \mathrm{~mJ} /$ pulse for free running with a long pulse width of $1 \mathrm{~ms}$ to increase the lifetime of the flashlamp, and a Q-switched peak power pulse of 400 to $500 \mathrm{~kW}$. In the following subsections the design of the laser subsystems will be explained. The material parameters and

\begin{tabular}{|c|c|c|}
\hline Symbol & Quantity & Value \\
\hline$T_{o}$ & $\begin{array}{l}\text { Initial transmission of } \\
\text { saturable absorber }\end{array}$ & $50 \%$ \\
\hline$R$ & Output coupler reflectance & $50 \%$ \\
\hline$n_{g s}$ & Saturable absorber atoms density & $1.6 \times 10^{24} \mathrm{~m}^{-3}$ \\
\hline$l_{s}$ & Saturable absorber thickness & $5 \mathrm{~mm}$ \\
\hline$(L \times d)$ & Gain medium dimensions & $5 \mathrm{~cm} \times 5 \mathrm{~mm}$ \\
\hline$E_{p}$ & Pump energy & $22.5 \mathrm{~J}$ \\
\hline$t_{p}$ & Pump energy pulse width & $1 \mathrm{~ms}$ \\
\hline$V$ & Charging voltage & $310 \mathrm{~V}$ \\
\hline$\sigma_{s}$ & Slope efficiency & $1.2 \%$ \\
\hline$E_{\text {out }}$ & Free running laser output energy & $150 \mathrm{~mJ}$ \\
\hline$E_{Q}$ & Q-switched laser pulse energy & $52 \mathrm{~mJ}$ \\
\hline$f$ & Pulse repetition rate & $0.1 \mathrm{~Hz}$ \\
\hline
\end{tabular}
the design parameters obtained from the simulation model of the realized passively Q-switched Nd:YAG laser are listed in Table 1

Table 1: Design parameters of the passively Q-switched ND:Yag laser 


\section{A. Design and implementation of the pump unit}

The simplicity of this laser system comes from using simple, cheap and effective components of the power supply and the pump cavity. We avoid using any transformers in the pump unit, whether it was for charging or triggering circuit. In the charging circuit, the input is 220 VAC passed through full wave rectifier to be DC $310 \mathrm{~V}$, which is sufficient voltage for many applications. In the triggering circuit, we did not use a triggering transformer and its associated electronics are required to control the triggering circuit of the flashlamp. Instead, we used a simple piezoelectric transducer where the mechanical shock is converted to an electrical signal which could effectively provide with the required spark to ionize the gases of the flashlamp. The result is a very simple, light and effective pumping unit for the proposed Q-switched Nd:YAG laser. From our enhanced laser mathematical model and simulation tool, it was found that the pump energy required to obtain the required laser output is $E=22.5 \mathrm{~J}$, the charging voltage $V_{\mathrm{o}} \approx 310 \mathrm{~V}$ and the pulse width of $t_{p}=1 \mathrm{~ms}$, a single capacitor has capacitance of $\mathrm{C}=470 \mu \mathrm{F}$, and inductance $L_{i n}=236 \mu \mathrm{H}$ (measured by an L-C meter) are used. The used flashlamp has $3 \mathrm{~mm}$ bore diameter and $5 \mathrm{~cm}$ length. Fig. 1 shows a schematic diagram of the designed flashlamp power supply. The triggering voltage of the flashlamp is supplied by a simple commercial piezoelectric transducer to avoid using complicated triggering circuit. The triggering circuit creates an ionized spark streamer between two electrodes so that the main discharge can occur. A $0.3 \mathrm{~mm}^{2}$ nickel wire is wrapped around the flashlamp for easy triggering. Simulation and layout of the pump circuit is implemented using OrCAD.

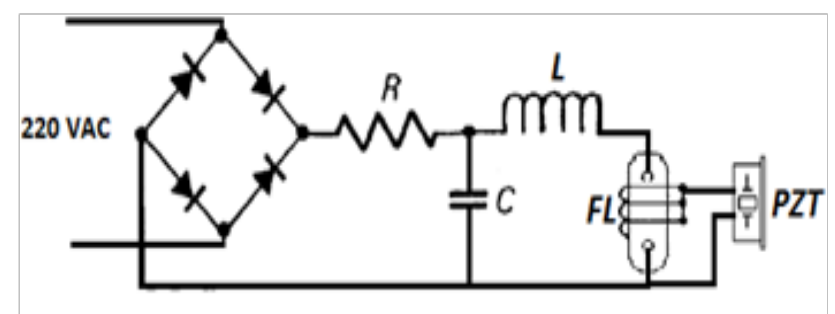

Fig. 1: Schematic diagram of the pumping unit of the proposed laser system.

\section{B. Optical resonator}

Plane parallel mirrors resonator were used as it provides easy and compact design, enables the use of the laser rod volume and provide large beam diameter approximately it is equal to the laser rod diameter $5 \mathrm{~mm}$ and this provide low divergence angle. The mirrors separation was $20 \mathrm{~cm}$. Two dielectrically coated flat mirrors were used. The rear mirror is totally reflecting mirror and the front mirror has a reflectivity of $50 \%$, each of diameter $25.4 \mathrm{~mm}$. Both gain medium and passive Q-switch are antireflection (AR) coated at $1064 \mathrm{~nm}$. All the resonator surfaces of the rod, Q-switch, and mirrors are flat.

\section{Design of the pump cavity}

In designing a pump cavity, the main objective is to maximize the amount of light directed from the flash lamp to the Nd:YAG rod. High reflectivity of the cavity walls at the absorption band of the laser material is required. There are many pump cavity configurations but the most commonly used are the elliptical pump and the closedcoupled cavities. In the elliptical pump cavities, the laser rod and the lamp are placed at the focal points of the ellipse to maximize the amount of lamp light directed to the rod. In the closed-coupled configuration, the lamp is placed as close as possible to the rod. In this case, the radiation directed from the lamp to the rod contributes more than just reflected radiation from the cavity walls to the rod. Elliptical pump cavities need careful design and implementation, to allow all of the light to correctly imaged into the rod, otherwise thermal losses will increase. These losses can be minimized by using a closed-coupled cavity. This approach allows more symmetric pumping and increasing beam quality. For easier implementation, an oval-shaped single-lamp closed-coupled configuration was designed. The cavity material was chosen to provide low cost and high mechanical strength, low thermal expansion, high thermal conductivity, and we found that the copper is the best material for this purpose. The copper cavity is nickel plated and is polished to a mirror surface. Nickel material is used to provide a hard surface which polishes very easily, and when plating on copper is much more durable than on other cavity materials. The rod and the flashlamp were fixed at the end of the cavity using silicon O-rings. The design of the pump cavity is implemented using inventor professional. Fig. 2 shows the designed oval shaped single-lamp closed-coupled cavity and its dimensions.
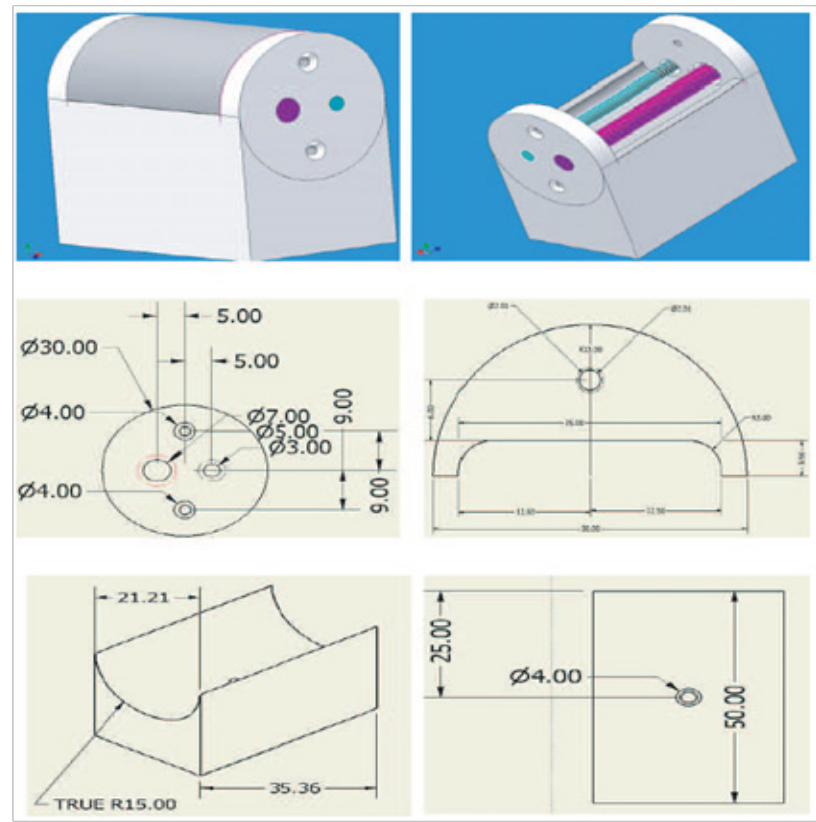

Fig. 2. Oval shaped single-lamp closed-coupled cavity design using inventor professional. 
D. Realization of the passively Q-switched Nd:YAG laser

Based on the design parameters obtained using the enhanced mathematical model, the passively Q-switched pulsed Nd:YAG laser is built and tested. Fig. 3 shows a photograph of the realized passively Q-switched pulsed $\mathrm{Nd}$ :YAG laser.

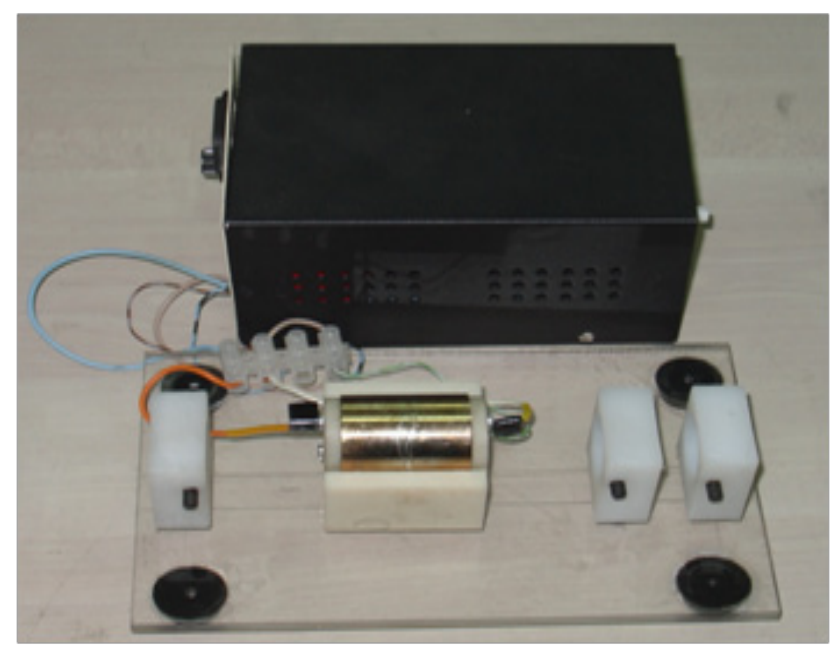

Fig. 3: Photograph of the overall realized passively Q-switched Nd:YAG laser.

\section{E. Measurement and analysis}

The laser output energy of the free running and Q-switching modes of the realized passively Q-switched Nd:YAG laser was measured. The used measurement tools are high voltage probe, voltmeter, ultrafast photodiode, digital oscilloscope, energy meter, and IR viewer. Fig. 4shows the measured free running laser output pulse has an energy of $150 \mathrm{~mJ}$ and pulse width of $1 \mathrm{~ms}$.

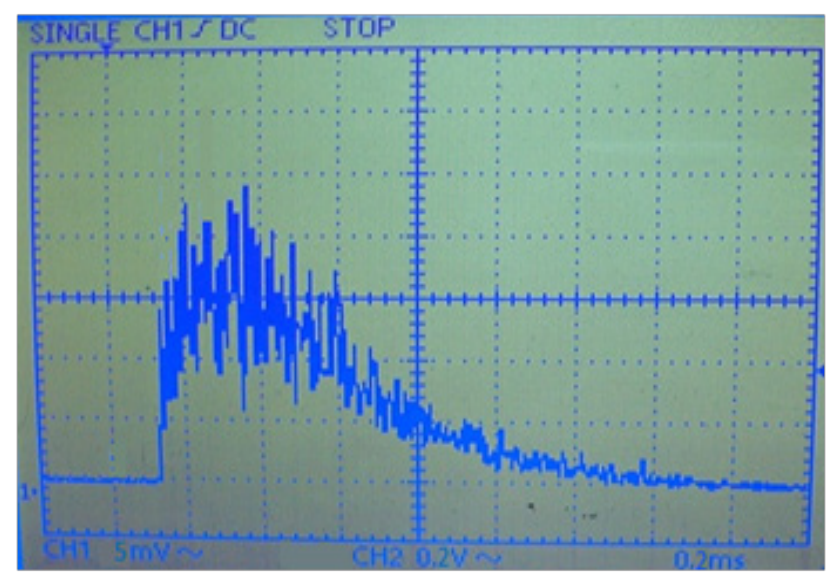

Fig. 4: The measured free running laser output pulse.

Fig. 5 shows the measured single shot Q-switched laser output that consists of a main pulse, followed by a train of 5 secondary pulses having different amplitudes and pulse widths, however, it has been found that the number and amplitude of the secondary pulses vary slightly from shot to shot (3-8 pulses). The amplitude of the main pulse was always the largest and most stable. We can attribute the change of the amplitudes and the number of secondary pulses to the nonlinearity of the passive q-switch crystal, the tolerance of the power supply of the flash lamp causes a slight change of the pump energy or due to the change in the environmental conditions when we performed the measurements in different days.

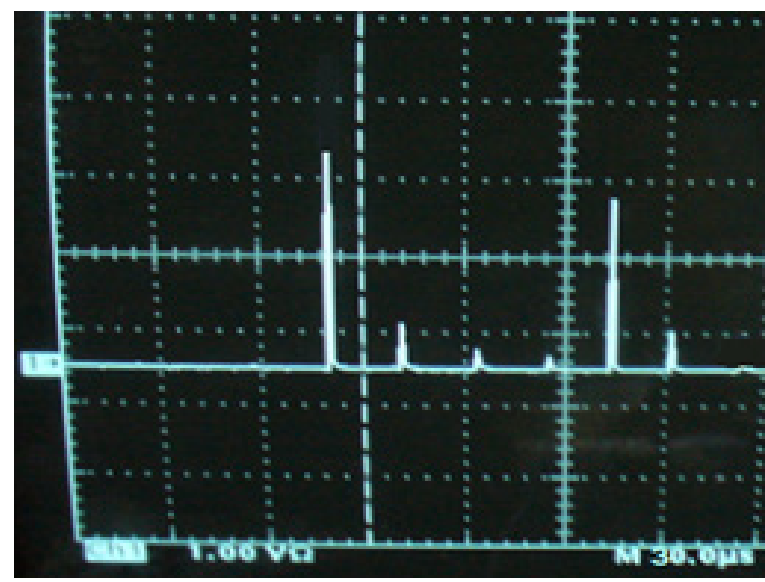

Fig. 5: Q-Switched pulse packet (30 $\mu$ s/div).

The maximum total output pulse energy of the 6 pulse train is $52.4 \mathrm{~mJ}$ distributed as follows : $22 \mathrm{~mJ}, 4.8 \mathrm{~mJ}, 2 \mathrm{~mJ}$, $1.6 \mathrm{~mJ}, 18 \mathrm{~mJ}$, and $4 \mathrm{~mJ}$, respectively, the order is as shown in Fig. 5. The average total duration of the output pulse train is $120 \mu \mathrm{s}$. The main pulse has been further expanded and is shown in Fig. 6. From this figure, the pulse width of the main Q-switched pulse at the FWHM is approximately $47 \mathrm{~ns}$ with peak power of $468 \mathrm{~kW}$.

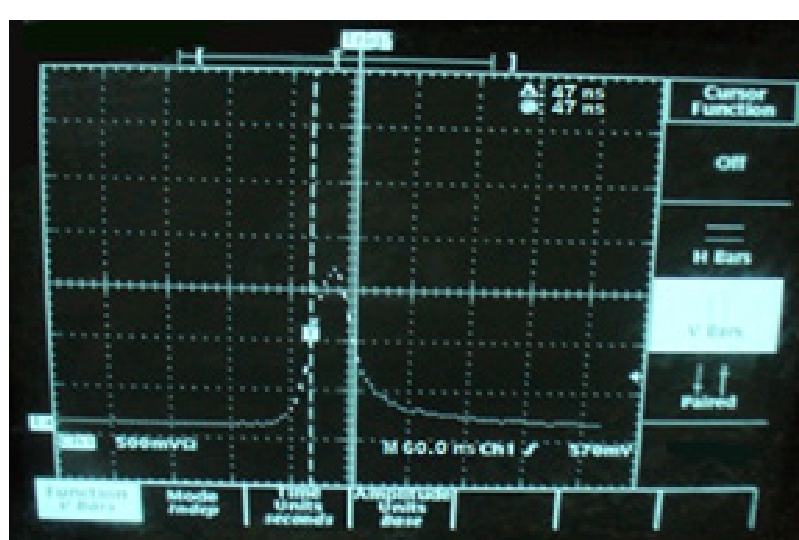

Fig. 6: Resolution of the main pulse in the Q-switched pulse packet at expanded time scale $(60 \mathrm{~ns} / \mathrm{div})$.

The measurements show that the realized system achieves the required free running output pulse energy, pulse width, and the Q-switched output peak power. During the realization of this laser system, we found out that generally the simulation results are in good agreement with the measured results. However, the measured and the simulation results sometimes are not exactly the same, 
this may be due to that the practical systems depends on the quality of system manufacturing, tolerance and accuracy of the electronic or optical components, inherent variations caused by operational use and alignment of the optical system. Although it is simple, cheap and compact construction, the proposed laser system can be used in many applications such as Laser-induced breakdown spectroscopy (LIBS), LIDAR, laser target designation, range finding and some medical applications. The proposed laser system can be portable by modifying the power supply to operate with batteries.

\section{CONCLUSION}

We have successfully realized a multipulse passively Q-switched pulsed Nd:YAG laser system for defense applications. A modified simulation model that can accurately predict the dynamic behavior of passively Q-switched solid-state lasers with $\mathrm{Cr}^{4+}$ :YAG as saturable absorber is used to extract the design parameters of the proposed laser system. The proposed system provides a potential compact and portable passively Q-switched laser source that can be easily used in the field operations. The system consists of a $5 \mathrm{~cm} \times 5 \mathrm{~mm} \mathrm{Nd}$ :YAG laser rod, pump energy of $22.5 \mathrm{~J}$, pulse width $1 \mathrm{~ms}$, cavity length $20 \mathrm{~cm}$, output coupler reflectance 50\%, a linear Xenon flash lamp, a closed-coupled cavity, $\mathrm{Cr}^{4+}$ :YAG as saturable absorber with initial transmission of $50 \%$. We obtained a single shot Q-switched laser output that consists of a train of 6 pulses. The maximum total output pulse energy of the pulse train is $52.4 \mathrm{~mJ}$ with the main Q-switched pulse has energy of 22 $\mathrm{mJ}$, pulse width of $47 \mathrm{~ns}$ and peak power of $468 \mathrm{~kW}$.

\section{REFERENCES}

[1] D. Kracht, S. Hahn, R. Huss, J. Neumann, R. Wilhelm, M. Frede and P. Peuser, "High efficiency, passively Q-switched Nd:YAG MOPA for spaceborne laser-altimetry," Proc. SPIE, pp. 548-555, 2006

[2] M. E. Jazi, M. D. Baghi, M. Hajimahmodzadeh, and M. Soltanolkotabi, "Pulsed Nd:YAG passive Q-switched laser using Cr4+:YAG crystal," Opt. Laser Technol., vol. 44, pp. 522-527, 2012.

[3] W. Koechner, "Solid-state laser engineering," 6th ed.New York, USA, Springer, 2006

[4] A. A. Mohamed and S. S. A. Ghoniemy, "Enhanced Passively Q-switched Nd:YAG Laser Modeling," in 26th National Radio Science Conference (NRSC2009), Egypt, 2009.

[5] J. J. Degnan, "Optimization of passively Q-Switched lasers," IEEE J.Quantum Electronics, vol. 31, pp. 1890-1901, 1995. 